\title{
Propuesta Metodológica para la Construcción de Objetos Virtuales de Aprendizaje basados en Realidad Aumentada
}

\author{
Luis C. Tovar, José A. Bohórquez y Plinio Puello \\ Universidad de Cartagena, Facultad de Ingeniería, Grupo de Investigación en Tecnologías de las \\ Comunicaciones e Informática, GIMATICA, Avenida del Consulado, Calle 30, No. 48 - 152, Cartagena- \\ Colombia. (e-mail: Itovarg@unicartagena.edu.co, jabohorqueza@gmail.com, \\ ppuellom@unicartagena.edu.co ).
}

Recibido Oct. 1, 2013; Aceptado Nov. 12, 2013; Versión final recibida Ene. 20, 2014

\begin{abstract}
Resumen
El objetivo del artículo es describir una metodología mixta para el desarrollo de objetos virtuales de aprendizaje basados en realidad aumentada, formada por la metodología de desarrollo de objetos virtuales de aprendizaje AODDEI (Análisis, Obtención, Diseño, Desarrollo, Evaluación, Implementación) y la ingeniería de software basada en componentes. Se determinaron las características de la metodología mixta con el fin de seleccionar los atributos que mejor se adaptaron a las tecnologías requeridas para implementar la realidad aumentada en dispositivos móviles. Se aplicó la metodología resultante a un caso de estudio y se desarrolló un objeto virtual de aprendizaje, mostrando la viabilidad funcional del procedimiento propuesto. Se concluye que el uso de la ingeniería de software basada en componentes permitió a la metodología AODDEI ser más versátil para la creación de objetos virtuales de aprendizaje basados en tecnologías emergentes.
\end{abstract}

Palabras clave: $A O D D E l$, ingeniería de software basada en componentes, objetos virtuales de aprendizaje, realidad aumentada, dispositivos móviles

\section{Methodological Proposal for the Construction of Virtual Learning Objects based on Augmented Reality}

\begin{abstract}
The objective of this paper is to describe a mixed methodology for the development of virtual learning objects based on augmented reality, which consists of the development methodology of virtual learning objects ACDDEI (Analysis, Collection, Design, Development, Evaluation and Implementation) and component based software engineering. The characteristics of the mixed methodology were determined with the aim of selecting the attributes that better adapted to the technologies required to implement the augmented reality on mobile devices. The resulting methodology was applied to a case study and a virtual learning object was developed, demonstrating the feasibility of the proposed methodology. It is concluded that the use of component based software engineering allowed ACDDEl is more versatile for creating virtual learning objects using emerging technologies.
\end{abstract}

Keywords: ACDDEl, component based software engineering, virtual learning objects, augmented reality, mobile devices 


\section{INTRODUCCIÓN}

Los rasgos políticos, culturales y económicos que caracterizan a la sociedad del siglo XXI, ha permitido el surgimiento de la llamada sociedad digital (Lévy, 2007). La cual se caracteriza por el uso dominante de las tecnologías de la información y comunicación (TIC) para el desarrollo de actividades tales como investigación, comunicación, compartir conocimiento e información, producir, organizarse y administrar (Bustos y Coll, 2010). Las TIC han llegado a revolucionar muchos aspectos de la cotidianidad de las personas y entre estas se encuentra la labor educativa, la utilización de las TIC potencia los métodos de enseñanza y agiliza los tiempos de apropiación del conocimiento, por lo cual las Universidades deben hacer uso de las mismas para formar profesionales competentes, que estén a la par de las comunidades académicas de este mundo globalizado (Gerrero V., 2004). Por lo que realizar herramientas como apoyo al proceso enseñanza-aprendizaje, ha sido el reto a lo largo del tiempo en la educación mundial, desde la educación básica primaria hasta la educación superior, constantemente en la búsqueda de métodos para propiciar la participación activa del estudiante en el proceso.

Algunas de las tecnologías que hacen uso de los recursos que brindan las TIC, son los dispositivos móviles y la realidad aumentada (RA), las cuales permiten crear contenidos educativos capaces de apoyar el proceso de enseñanza-aprendizaje, como lo son los Objetos Virtuales de Aprendizaje (OVA'S), los cuales son estructuras organizadas y diseñadas por equipos multidisciplinarios que pueden usar las ventajas que brinda la RA para captar la atención del público al cual va dirigida la enseñanza. Esta investigación se centró en la formulación de una metodología mixta para la construcción de OVA'S, debido a que las metodologías existentes están diseñadas para la creación de estructuras tipo páginas web y software de escritorio, por lo cual se generaban inconvenientes de acoplamiento al momento de desarrollar OVA'S haciendo uso de tecnologías emergentes como la RA en dispositivos móviles. Lo cual representa la importancia de la metodología propuesta en el presente artículo.

El auge de la educación virtual, y por ende de los contenidos digitales que se pueden realizar gracias a esta, puso el centro de las miradas en el desarrollo de materiales educativos. Inicialmente fueron las organizaciones empresariales las que hicieron uso de la educación virtual con el fin de capacitar de una manera más eficiente a sus empleados, quienes en ocasiones se encontraban en lugares lejanos y distribuidos, por lo cual se vieron en la necesidad de usar metodologías flexibles que permitieran optimizar la creación de materiales educativos, en cuanto a tiempo, diseño y distribución (Astudillo y Willging, 2011). El término objetos de aprendizaje (OA) fue utilizado por primera vez en 1992 por Wayne, el cual fue inspirado al ver a sus hijos jugar con unos bloques de LEGO® y los asoció con bloques de aprendizaje normalizado que podían ser reutilizados en el proceso educativo (Hodgings, 2000).

Durante 1994 y 1995 la empresa Oracle comienza a desarrollar Oracle Learning Application (OLA) que fue un intento por crear un software propietario para diseñar materiales a través de OA. El proyecto de Oracle no prosperó, pero Tom Kelly y Chuck Barritts, responsables del proyecto en Oracle, lo continuaron en Cisco System y, en 1999, presentan Reusable Learning Objects (RLO). Un RLO se creaba combinando una vista, un resumen, una evaluación, y entre 5 y 7 objetos informativos reutilizables (RIO, por sus siglas en inglés). Estos a su vez estaban compuestos por contenidos, actividades y evaluaciones (Cisco Systems, Inc, 1999).

En los años posteriores el concepto de objeto de aprendizaje experimentó cambios y aún no se había establecido una definición de manera universal, por lo que variaban dependiendo del autor. Para Dowenes (2001), los OA son herramientas educativas que utilizan las TIC con el fin de lograr contenidos amigables y sustanciosos para las personas que hacen uso de ellos. Para el Ministerio de Educación de Colombia(2007) un $\mathrm{OA}$ es un conjunto de recursos digitales que pueden ser utilizados en diversos contextos, con un propósito educativo y constituido por al menos tres componentes internos: contenidos, actividades de aprendizaje y elementos de contextualización. Además, el objeto de aprendizaje debe tener una estructura de información externa (metadato) para facilitar su almacenamiento, identificación y recuperación. Este es un mediador pedagógico que ha sido diseñado intencionalmente para un propósito de aprendizaje y que sirve a los actores de las diversas modalidades educativas (Colombia Aprende, 2008).

Para optimizar la creación de los OVA'S se han diseñado varias metodologías que apoyan el proceso, entre las que se encuentran Medhime 2.0, una metodología para el desarrollo de Objetos Virtuales de Aprendizaje en plataformas libres tipo Moodle, basada en el estándar SCORM, esta metodología posee 4 etapas las cuales son: análisis del dominio, diseño conceptual, diseño navegacional y diseño comunicacional (Sirvente, 2011). Ha sido usada en numerosos proyectos como apoyo al proceso educativo en las aulas de clases, en áreas de la informática, matemáticas, sociales, biología entre otras ramas del saber. Algunos de estos proyectos son: Cola de Prioridad Hipermediada con Medhime 2.0 (Correa y Bordon, 2011); Números Naturales (Alamo y Gutierrez, 2012); Investigación Operativa en Educación a Distancia (Mattar y Pacheco, 2012). 
En la Universidad del Valle se creó otra metodología de diseño de Objetos de Aprendizaje, la cual es producto de la combinación de un modelo pedagógico y un modelo de ciclo de vida de desarrollo de software. Está constituida por 5 fases a saber: formulación y planificación, análisis, ingeniería, generación de páginas y pruebas, y evaluación del cliente (Borrero et al., 2010). Esta metodología ha sido usada en el diseño de objetos de aprendizaje a nivel de especialización y maestría tales como enfermería, ingeniería eléctrica y electrónica, ingeniería civil y psicología. Algunos de los OVA's son: Repuesta Sísmica de las Edificaciones (Vargas, 2010); Sistemas digitales, conceptos básicos (Arteaga y Velazco, 2007); Redes neuronales artificiales, conceptos básicos, arquitectura y aprendizaje (Caicedo y López, 2007).

Por otra parte, AODEEI, es una metodología para desarrollar OVA'S e integrarlos a un sistema de gestión de aprendizaje teniendo en cuenta las fases de análisis, obtención, diseño, desarrollo, evaluación e implementación. Esta metodología tuvo su primera aplicación en un curso intensivo de la unidad de formación de profesores en la Universidad Autónoma de Aguascalientes, con un desempeño sobresaliente en su aplicación (Álvarez et al., 2006).

La educación soportada en la tecnología no solo se ha visto influenciada por el desarrollo de los conceptos de OA'S o el surgimiento de nuevas metodologías, también ha experimentado cambios como consecuencia de los avances tecnológicos, los cuales también se ven reflejados en los OVA'S. Con el advenimiento de Internet, surge un nuevo concepto relacionado con las telecomunicaciones como lo son la tele-educación, el aprendizaje basado en la web y como punto final un esquema que formalizo los anteriores llamado ELearning, el cual hace uso de herramientas multimedia, internet y tecnologías de la web con el fin de soportar el proceso enseñanza-aprendizaje. Por otra parte, el auge de la tecnología móvil, ha dado lugar al Mobile Learning o M-Learning, que es definido como la difusión de contenidos formativos a través de dispositivos móviles (Flétscher y Morales, 2007). Según Ramírez (2008), M-Learning busca fortalecer las capacidades de interacción y apoyo en el proceso de enseñanza-aprendizaje, y las capacidades de comunicación en los distintos procesos del modelo educativo. Para el año 2010, esta evolución ha permitido enriquecer el M-Learning con muchas tecnologías, por ejemplo la RA (De La Torre et al., 2013). La RA permite al usuario ver el mundo real con objetos virtuales superpuestos o compuestos con el mundo real (Azuma, 1997). Los objetos virtuales pueden ser manipulados por las personas, las cuales los coordinan para obtener el punto de vista que deseen (Martin, 2010). La RA se encuentra en desarrollo, pero al mismo tiempo se ha ido generando un impacto positivo sobre el mercado, gracias a la cantidad de proyectos funcionales que se han y siguen realizando utilizando esta tecnología (Carrasquilla et al., 2011).

El hacer uso de los dispositivos móviles y la RA como herramientas en el proceso de enseñanzaaprendizaje y más concreto en el desarrollo de OVA'S genera una serie de inconsistencias con las metodologías de OVA'S mencionadas anteriormente, ya que la mayoría están diseñadas para generar contenidos tipo páginas web y software de escritorio y no tienen en cuenta el uso de tecnologías emergentes como lo son la RA y los dispositivos móviles. Es importante aclarar que las metodologías expuestas han obtenidos resultados favorables durante su aplicación y han demostrado su utilidad. Pero tienen un enfoque diferente, por lo cual no se ajustan de manera adecuada a las tecnologías que se desean utilizar, razón por la cual se presenta la siguiente metodología mixta como respuesta al inconveniente mencionado.

\section{METODOLOGÍA MIXTA PROPUESTA}

Con el fin de establecer una metodología capaz de desarrollar OVA'S utilizando tecnologías emergentes como lo es la RA en dispositivos móviles, se mezclaron la AODDEl para el desarrollo de OVA'S y la ingeniería de software basada en componentes (ISBC), seleccionando las características respectivas que mejor se adaptaron.

De AODDEI se utilizaron 5 fases, de la primera fase fueron útiles todos los pasos 1,2 y 3 (Análisis, obtención y digitalizar el material), debido a que fue necesario establecer las bases de los OVA'S y los contenidos que se presentarían en este. De la fase 2 se usó el paso 4 (Arme la estructura del OVA), en el cual se estableció la manera como serian diseñados los OVA'S. De la fase 3 , solo fue útil el paso 5 (Armado), en el cual se ensamblaron todos los componentes de los OVA'S obtenidos en las fases anteriores. Los pasos 6 y 7 (Empaquetar y almacenar el OVA en un repositorio temporal) de la fase 3 son utilizados para empaquetar y almacenar OVA'S orientados a software de escritorio y páginas web, cosa que no es el objetivo de esta investigación por lo cual se omiten. En la fase 4, solo se usó el paso 8 (Evaluar el OVA) el cual permitió examinar la pertinencia de los OVA'S. El paso 9 (Almacenar el OA en un repositorio de OVA'S evaluados) plantea almacenar los OVA'S en un repositorio de OVA'S evaluados, el cual se omitió con el fin de hacer una sola publicación final en la fase 5 llamada implantación. 
De la ingeniería de software basada en componentes (ISBC), se utilizaron las 5 fases, las cuales son Comunicación con el cliente, Planificación, Análisis de riesgos, Construcción y adaptación de los componentes de ingeniería y Evaluación del cliente. Se pudo haber usado otras metodologías de desarrollo de software como la propuesta por Borrero (2010), sin embargo se escogió ISBC porque hace que no sea necesario comenzar desde cero el desarrollo de los OVA'S, especialmente bajo el enfoque de la RA y la herramienta de desarrollo seleccionada, donde se conjuga la interacción de diferentes componentes como se describe a continuación

El proceso lo inicia la cámara del dispositivo móvil. La cámara envía cada captura o frame de las imágenes que son tomadas por la misma, estos Frame son enviados al componente Pixel Format Conversión (Convertidor de Formato de Pixel) que convierte la imagen capturada a un formato compatible con OpenGL ES 2.0, posteriormente es enviado al Tracker (Seguidor), este componente posee un conjunto de algoritmos encargados de validar y comparar los Frame con las Imágenes o Marcadores que se encuentran en Device Database (Base de Datos en Dispositivo) o Cloud Database (Base de Datos en la Nube) según sea el caso, luego el siguiente paso es enviar la información al video background renderer quien determina si la imagen capturada corresponde a un marcador, si esto es correcto este componente renderizará los modelos 3D en la pantalla del dispositivo móvil.

En resumen, la metodología AODDEI es usada como base para la formación de la estructura del OVA y se seleccionó entre las expuestas en el apartado anterior debido a que presenta la estructura metodológica más atractiva para la investigación y permite adaptarse con facilidad a las fases de la ISBC. Esta última, aunque puede ser usada en cualquier desarrollo de software, permite complementar la metodología AODDEl en cada una de las fases para la conformación de los OVA'S, además que permite el uso de componentes para la implementación de la realidad aumentada que ya se encuentran desarrollados, los cuales pueden ser reutilizados y aplicados para cumplir los objetivos propuestos en un lapso menor de tiempo. Estas metodologías permitieron construir OVA'S utilizando tecnologías emergentes, lo cual se presenta como reto e innovación para la investigación. En la siguiente tabla se encuentra de manera explícita la forma como ambas metodologías fueron entrelazadas:

Tabla 1: Fases de AODDEI, fases de ISBC y la metodología mixta final propuesta

\begin{tabular}{|l|l|l|l|}
\hline \multicolumn{1}{|c|}{ POSOS } & \multicolumn{1}{c|}{ ISBC } & \multicolumn{1}{c|}{ MIXTA } \\
\hline $\begin{array}{l}\text { 1:Análisis y } \\
\text { obtención }\end{array}$ & $\begin{array}{l}\text { 1: Análisis } \\
\text { 2: Obtención del material }\end{array}$ & Comunicación con el cliente & Análisis del negocio \\
\hline 2: Diseño & $\begin{array}{l}\text { 4: Armado de la estructura del } \\
\text { OVA }\end{array}$ & $\begin{array}{l}\text { Planificación } \\
\text { Análisis de riesgos }\end{array}$ & $\begin{array}{l}\text { Diseño e Identificación de } \\
\text { herramientas }\end{array}$ \\
\hline 3: Desarrollo & Paso 5: Armado & $\begin{array}{l}\text { Construcción y adaptación de } \\
\text { los componentes de ingeniería }\end{array}$ & $\begin{array}{l}\text { Construcción y adaptación de } \\
\text { los componentes de ingeniería }\end{array}$ \\
\hline $\begin{array}{l}\text { 4: Evaluación } \\
\text { 5: Implantación }\end{array}$ & $\begin{array}{l}\text { 8: Evaluación del OVA } \\
\text { 10: Integrar el OVA a un } \\
\text { sistema de gestión de } \\
\text { aprendizaje }\end{array}$ & Evaluación del cliente & Evaluación e implantación \\
\hline
\end{tabular}

Fase 1. Análisis del negocio:

En esta fase el experto en la temática junto con el equipo multidisciplinario, diseñador e ingeniero de sistemas se reúnen y definen los siguientes aspectos concernientes a los OVA'S:

Análisis:

La problemática a solucionar con el desarrollo de los OVA'S.

El público al que está dirigido el proyecto, en este caso es el mismo al que afecte el problema a solucionar.

La solución propuesta a la problemática y la temática que se abordará.

Las características básicas de los OVA'S a desarrollar.

Obtención:

Los requerimientos funcionales y no funcionales de los OVA'S basados en la manera como el experto aborda la temática. Se obtiene el inventario de los modelos 3D a desarrollar, los cuales son parte de los elementos multimedia inmersos en los OVA'S.

Digitalizar el material:

Se generan los materiales multimedia y los modelos 3D, que harán parte de los OVA'S. 
Los materiales multimedia y modelos $3 \mathrm{D}$ son validados por el experto en la temática, en caso tal de necesitar correcciones, éstas son realizadas de inmediato, preferiblemente antes de pasar a la siguiente fase.

\section{Fase 2. Diseño y selección de herramientas:}

En esta fase se debe diseñar la relación de los objetivos, los contenidos informativos, las actividades y la evaluación, como parte del diseño en la estructura de los OVA'S. también se deben identificar las herramientas a utilizar basándose en un análisis detallado.

Diseño: Se realiza la organización de los contenidos inmersos en los OVA'S. Tomando como punto de partida que los OVA'S buscan apoyar la apropiación del conocimiento en una temática especifica. Estos contenidos van organizados de la siguiente manera:

Contenidos informativos: Indicar la manera como será presentada la información, la navegabilidad y su organización.

Actividades: Indicar las actividades que se realizarán en los OVA'S con el fin de apoyar la apropiación de los conceptos presentados.

Evaluación: Se realiza con el fin de medir el nivel de apropiación de los conceptos expuestos en los OVA'S. Identificación de herramientas: En este apartado se debe realizar una exhaustiva investigación sobre las herramientas y componentes de realidad aumenta que mejor se adapten a las características del proyecto.

Análisis de herramientas: Se deben establecer prioridades dentro de las características de las herramientas, las cuales serán vitales para el desarrollo de los OVA'S, con el fin de llegar a la herramienta que mejor se adapte a las necesidades.

Fase 3. Construcción y adaptación de los componentes de ingeniería:

En esta fase se deben realizar los marcadores de realidad aumentada correspondiente a cada OVA'S. Además se lleva a cabo el desarrollo de la aplicación que corresponde a la formación de los OVA'S como tal, utilizando cada uno de los elementos generados en las fases anteriores como los modelos 3D, contenidos teóricos, contenidos evaluativos, audios y marcadores.

Construcción de los marcadores: En esta instancia se realizan los marcadores de realidad aumentada que serán útiles para cada OVA. Para lo cual se debe tener en cuenta características importantes que garanticen el diseño apropiado de los marcadores como la cantidad de vértices, colores, contrastes, entre otros.

Construcción de la aplicación: Esta es una de las partes más importantes en el desarrollo de los OVA'S ya que aquí se hace uso de los componentes seleccionados en fases previas para integrar todos los elementos constitutivos de los OVA'S, tales como los modelos 3D, archivos multimedia, contenidos informativos, actividades y evaluación.

\section{Fase 4. Evaluación e implantación:}

En esta fase se realiza el proceso de evaluación a los OVA'S. Primero bajo la supervisión del personal calificado, tomando como base los requerimientos funcionales y no funcionales. Luego por el público al cual van dirigidos los OVA'S, en esta instancia se pueden aplicar encuestas, actas u otras actividades que sirvan como evidencia del proceso.

Evaluación por personal calificado: En esta instancia se colocan los OVA'S a disposición del personal experto en la temática para comprobar que cumplen con las características deseadas. Se tiene en cuenta el cumplimiento de los requerimientos funcionales y no funcionales. En caso de presentar correcciones deben ser atendidas antes de pasar a la siguiente instancia. Se recomienda obtener evidencia de este proceso.

Evaluación estudiantes: En este paso es importante que los OVA'S ya estén avalados por el personal calificado, luego son evaluados por los estudiantes haciendo uso de ellos, verificando su utilidad en el proceso de aprendizaje e indicando posibles mejoras de acuerdo a la necesidad. Se recomienda tener soportes como evidencia en este proceso. 
Implantación: En esta fase se realiza el proceso de publicación para que los usuarios tengan acceso a los contenidos de los OVA'S, por lo cual se deben integrar los OVA'S a un sistema de gestión de aprendizaje que facilite su acceso, como Moodle u otra plataforma con este fin. Además, los OVA's pueden ser distribuidos para los diferentes sistemas operativos móviles mediante una app en sitios como App Store o Play Store.

\section{CASO DE ESTUDIO}

Al aplicar la metodología mixta al desarrollo de OVA'S para el estudio de la anatomía de órganos dentales en la Facultad de Odontología de la Universidad de Cartagena Colombia se obtuvieron los siguientes resultados de acuerdo a las fases propuestas anteriormente:

\section{Fase 1: Análisis del negocio.}

Por medio de la ejecución de esta fase se logró obtener los siguientes resultados:

Se definió la problemática a saber: falta de ayudas tecnológicas como apoyo a la enseñanza de la anatomía dental para los estudiantes de tercer semestre en el pregrado de odontología de la Universidad de Cartagena.

Se propone como solución la implementación de OVA'S, utilizando tecnologías como la realidad aumentada, el modelado 3D y los dispositivos móviles.

Se definieron las características básicas que debían tener los OVA'S, para la presentación de la temática. A través de una serie de reuniones y entrevistas se establecieron los requerimientos funcionales y no funcionales que debían manejar los OVA'S. Se obtuvo el inventario de las piezas dentales a modelar, el cual corresponde a los 16 órganos dentales que estarían inmersos en los OVA'S. Se generaron cada uno de los materiales multimedia y los modelos 3D representativos de las piezas dentales y a su vez estos pasaron por una exhaustiva evaluación hasta cumplir con las exigencias de los expertos en la temática, algunos de estos modelos se presentan en la figura 1 .
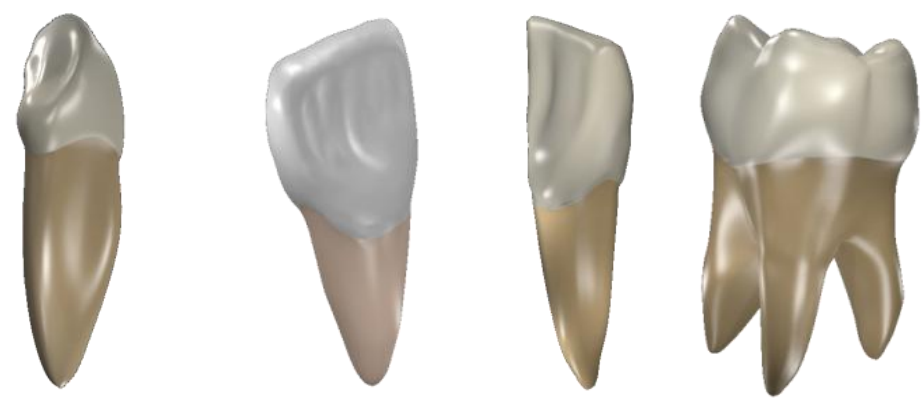

Fig.1: Modelos 3D

Fase 2: Diseño y selección de herramientas.

Esta fase reflejó los siguientes resultados:

Se diseñó la manera como estarían interrelacionados los contenidos informativos, actividades y evaluación que le permitirían a los OVA'S desempañarse como elementos de apoyo para la enseñanza de la temática anatomía dental.

Utilizando la experiencia de los autores en el área de RA, y siguiendo las descripciones provistas por (Lorett y Marrugo, 2011) y (Carrasquilla et al., 2011) se seleccionó la herramienta más apropiada para el desarrollo de los marcadores y la aplicación de realidad aumentada. Para ello se tuvo en cuenta la eficacia en la detección del marcador, la estabilidad, compatibilidad con modelos 3D de diferentes extensiones, el tipo de licenciamiento utilizado, la existencia de una comunidad para soporte, desarrollo multiplataforma, interacción con los modelos 3D y el permitir agregar contenido multimedia. Cada uno de estos aspectos se evaluó en cinco kits de desarrollo de software (SDK, por sus siglas en inglés) para realidad aumentada. Al final se seleccionó Qualcomm SDK® como el indicado para la investigación.

Fase 3: Construcción y adaptación de los componentes de ingeniería. 
En esta fase se obtuvo la creación de los marcadores para realidad aumentada correspondientes a cada OVA. Se construyeron los OVA'S utilizando cada uno de los elementos mencionados como resultados de las fases anteriores tales como: modelos 3D, marcadores, contenidos informativos, actividades, contenidos evaluativos. En las figuras 2 y 3 se puede observar la aplicación contenedora de OVA'S desarrollada:
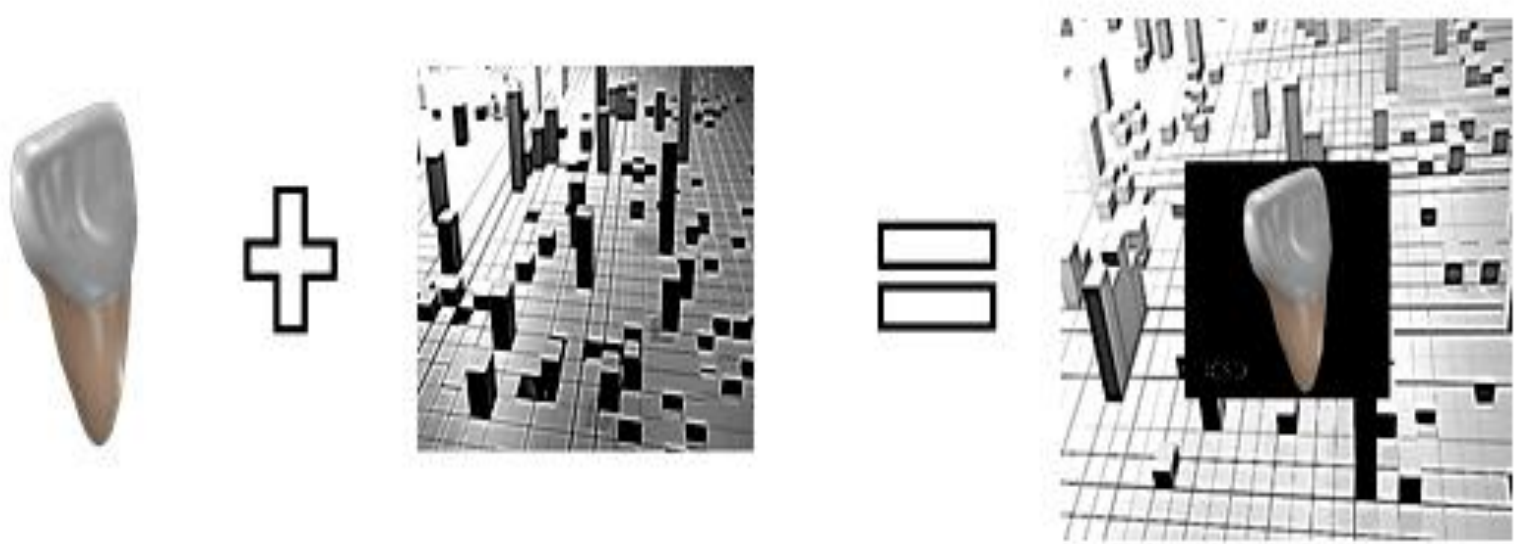

Fig.2: Creación de marcadores.

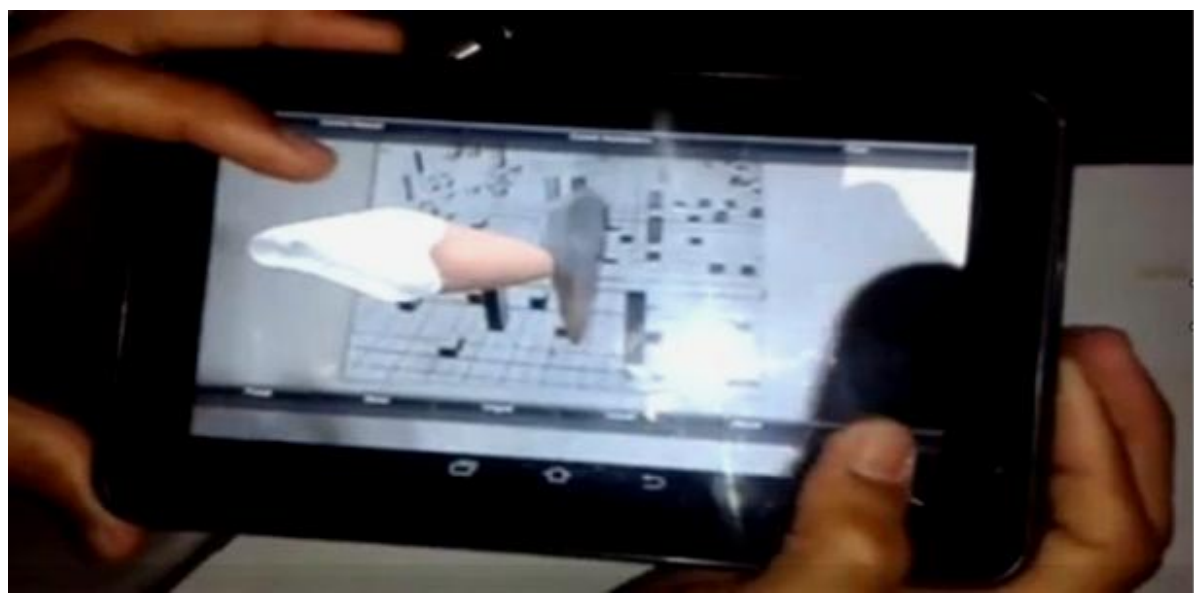

Fig.3: Aplicación en funcionamiento.

Fase 4: Evaluación e implantación.

Posterior a la creación de los OVA'S, estos pasaron por una serie de evaluaciones las cuales arrojaron los siguientes resultados:

El personal experto en la temática aprobó el funcionamiento general de la aplicación contenedora de OVA'S, por lo cual se pasó a la evaluación por parte de los estudiantes. Los estudiantes hicieron uso de los OVA'S, comprobaron su funcionamiento, los manipularon y a través de una encuesta realizada se concluyó que la aplicación AppTooth, es una herramienta que si apoya el proceso de enseñanza-aprendizaje de la anatomía dental.

Para la evaluación realizada a los OVA's por parte de los estudiantes de la Facultad de Odontología de la Universidad de Cartagena, se tomó una muestra significativa correspondiente al $40 \%$ de los estudiantes regulares de tercer semestre. La muestra seleccionada conserva las características más relevantes de la población, lo cual fue validado por el docente encargado. Los resultados de la evaluación de los estudiantes se resumen en los siguientes aspectos: 
El tiempo utilizado por la aplicación para la detección del marcador y la visualización de la pieza dental se midió teniendo en cuenta tres ítems para evaluación de la percepción de los estudiantes con respecto a este aspecto y se obtuvo que el $60 \%$ consideró la aplicación como rápida, el $40 \%$ norrnal y $0 \%$ lenta.

Los modelos 3D implementados corresponden a las piezas dentales reales. El $85 \%$ consideró que si, $15 \%$ dijo que no.

El contenido informativo fue considerado apropiado por el $85 \%$ y no por el $15 \%$. La evaluación con respecto al contenido informativo fue considerada pertinente por el $85 \%$ y no pertinente por el $15 \%$. El contenido evaluativo fue considerado de alta dificultad por el $7 \% ; 14 \%$ consideró que era de mediana dificultad y al $79 \%$ les pareció que tenía una dificultad baja. Se debe tener en cuenta que estos contenidos evaluativos fueron desarrollados con ayuda del docente y aplicados luego de que los estudiantes utilizaran los OVA's.

Nivel de satisfacción en el uso de la aplicación. 45\% dijeron estar muy satisfechos, frente al $55 \%$ que dijo estar satisfecho. Las otras dos opciones de evaluación eran insatisfecho y muy insatisfecho pero ningún estudiante seleccionó estas opciones. Los estudiantes que dijeron estar solamente satisfechos escogieron esa opción porque deseaban que el OVA fuera táctil, característica que posteriormente fue agregada.

El uso de la aplicación puede ayudar en el proceso enseñanza-aprendizaje de la temática. El 85\% consideró que si, frente al $15 \%$ que considera que no porque prefiere los dientes reales.

Posterior a las evaluaciones realizadas se pasó a publicar (implantación) la aplicación final en una plataforma contenedora de OVA'S, desde la cual se podrá acceder a los contenidos desarrollados. La plataforma contenedora también es resultado del grupo de investigación GIMATICA de la Universidad de Cartagena.

\section{CONCLUSIONES}

El articulo presentó la formulación de una metodología mixta para el desarrollo de OVA'S. Dicha metodología fue aplicada a un caso de estudio donde se desarrollaron una serie de OVA'S. Con base en los resultados obtenidos se concluye: La metodología propuesta permitió la consecución de los objetivos que planteaba el caso de estudio a lo largo de sus fases, lo que demuestra su viabilidad y su aplicación en otras investigaciones. La ISBC complementa a AODDEI volviéndola más versátil, debido a que no solo es funcional para ser aplicada en OVA'S tipo páginas web y software de escritorio sino que también puede ser usada para crear OVA'S que utilicen tecnologías emergentes como lo son la realidad aumenta en dispositivos móviles. Con la aplicación de esta metodología en el caso de estudio antes mencionado, es válido resaltar cómo los estudiantes asimilaron de una manera más didáctica y amena los contenidos de aprendizaje en OVA'S enfocados al M-Learning. Se destaca también que el uso de dispositivos móviles en el proceso de enseñanza-aprendizaje representa una tendencia novedosa, capaz de captar la atención del público joven.

\section{REFERENCIAS}

Álamo, G., y Gutierrez, A., "Números Naturales. MeDHiME 2.0, Metodología de Diseño Hipermedial de Materiales Educativos según el estándar SCORM", (en linea) (2012).. http://www.portalhuarpe.com.ar/medhime20/Talleres/Taller\%20Andalgala/04\%20Numeros\%20Naturales/nav egable/index.html. Acceso: Mayo (2013)

Álvarez, F., Osorio, B., Cardona, J., y Muñoz, J., "Objetos de aprendizaje integrados a un sistema de gestión de aprendizaje. Apertura Revista de Innovación Educativa", Vol. 6, Núm. 3, pp.109-117. (2006)

Arteaga, J., y Velazco, J., "Sistemas digitales. Conceptos básicos". Universidad del Valle. Cali, Colombia. (en linea). (2007). http://objetos.univalle.edu.co/?q=node/364. Acceso: Mayo (2013)

Astudillo, G. J., Sanz, C., y Willging, P.. "Análisis del estado del arte de los objetos de aprendizaje". Repositorio Institucional de la Universidad Nacional de la Plata. (en linea), (2011). http://sedici.unlp.edu.ar/bitstream/handle/10915/4212/Documento_completo.pdf?sequence=1. Acceso: Mayo (2013).

Azuma, R., "A Survey of Augmented Reality. Presence: Teleoperators and Virtual Enviroments", 6(4), pp. 355-385. (1997) 
Borrero, M., Cruz, E., Mayorga, S., y Ramírez, K., "Una metodología para el diseño de objetos de aprendizaje. La experiencia de la DINTEV de la Universidad del Valle". (2010) http://objetos.univalle.edu.co/files/articulo_AMED.pdf. Acceso: Agosto (2013)

Bustos, A., y Coll, C., "Los entornos virtuales como espacios de enseñanza y aprendizaje. Una perspectiva psicoeducativa para su caracterización y análisis". Revista Mexicana de Investigación Eductiva, 15( 44), 163-184. (2010)

Caicedo, E., y López, J., "Redes neuronales artificiales. Conceptos básicos, arquitectura y aprendizaje". Universidad del Valle, Cali, Colombia. (2007)

Carrasquilla, G.A., Pinilla, H., y Tovar, L.C., "Aplicacion de la Realidad Aumentada en la enseñanza de la simetria molecular para lograr un aprendizaje significativo". Informe de investigación, Universidad de Cartagena, Programa de Ingeniería de Sistemas. Cartagena, Colombia. (2011)

Cisco Systems, Inc. Cisco Systems Reusable. Cisco System (en linea) (1999). http://www.cisco.com/warp/public/779/ibs/solutions/learning/whitepapers/el_cisco_rio.pdf. Acceso: Mayo (2013)

Colombia Aprende. Nuevas Formas de Enseñar y aprender. (en línea), (2008). http://www.colombiaaprende.edu.co/html/directivos/1598/article-88892.html. Acceso: Abril (2012)

Correa, V., y Bordón, C., Cola de prioridad hipermediada con Medhime 2.0, MeDHiME 2.0, Metodología de Diseño Hipermedial de Materiales Educativos según el estándar SCORM, (en linea) (2011). http://www.portalhuarpe.com.ar/medhime20/Nuevos\%200A/Cola\%20de\%20Prioridades/Principal/ReloadCo ntentPreview.htm. Acceso: Mayo (2013)

De la Torre, J., Martin-Dorta, N., Saorín, J., Carbonell, C., y Contero, M., Entorno de aprendizaje ubicuo con realidad aumentada y tabletas para estimular la comprensión del espacio tridimensional. RED. Revista de Educación a Distancia, 37, 1-17. (en línea) (2013). http://www.um.es/ead/red/37/DELATORREetAL.pdf. Acceso: Agosto (2013)

Dowenes, S., Learning Objects: Resources For Distance Education Worldwide. International Review of Research in Open and Distance Learning, ISSN: 1492-3831 (en linea) 2(1). (2001). http://www.irrodl.org/index.php/irrodl/article/view/32/81. Acceso: Mayo (2013)

Flétscher, L., y Morales, Á., Modelo de desarrollo de servicios m-learning, una propuesta desde la concepción del servicio hacia la pedagogía. Revista Virtual Universidad Católica del Norte, 22, 1-22. (2007)

Gerrero, G., La Educación en el contexto de la Globalización. Revista Historia de la Educación Latinoamericana, 6( 6), 343-354. (2004)

Hodgings, W., Into the future: A vision paper. American Society of Training and Development (ASTD) and National Governors' Association (NGA) Comission on Technology and Adult Learning, p. 27. (2000)

Lévy, P., Ciberculura: la cultura de la sociedad digital. Mexico: Universidad Autónoma Metropolitana: ANTHROPOS. (2007)

Lorett, D. y Marrugo, T., INSITU, sistema de guia turístico insitu, basado en realidad aumentada para cartagena de indias. Tesis de Ingeniero, Universidad de Cartagena, Programa de Ingeniería de Sistemas. Cartagena, Colombia. (2011)

Martín-Gutierrez, J., Saorín, J., Contero, M., Alcañiz, M., Pérez-López, D., y Ortega, M., Design and Validation of an Augmented Reality for Spatial Abilities Development in Engineering Students. Computer \& Graphics, 34(1), 7-91. (2010)

Mattar, M., y Pacheco, V., Investigación Operativa en Educación a Distancia. MeDHiME 2.0, Metodología de Diseño Hipermedial de Materiales Educativos según el estándar SCORM, (en linea) (2012). http://www.portalhuarpe.com.ar/medhime20/Talleres/TALLERES\%20CUIM/Taller\%2010/T1006Investigacion-Operativa-en-Educacion-a-Distancia/navegable/index.html. Acceso: Mayo (2013) 
Ministerio de Educación Nacional Colombiano MEN. Catalogación de objetos de aprendizaje en instituciones de educación superior. (en línea), (2007). http://www.cvudes.edu.co/ModeloPedagogico/. Acceso: Marzo (2013)

Ramírez, M., Dispositivos de mobile learning para ambientes virtuales: implicaciones en el diseño y la enseñanza. Apertura Revista de Innovación Educativa, 8(9), . 82-96. (2008)

Sirvente, A., MeDHiME 2.0. Metodología para diseñar objetos de aprendizaje, migrando rápidamente a los docentes no informáticos. (en línea), (2011).

http://www.virtualeduca.info/ponencias2011/20/Medhime\%20virtualeduca.pdf. Acceso: Marzo (2013)

Vargas, G., Repuesta Sísmica de las Edificaciones. Universidad del Valle, Cali, Colombia. (2010) 